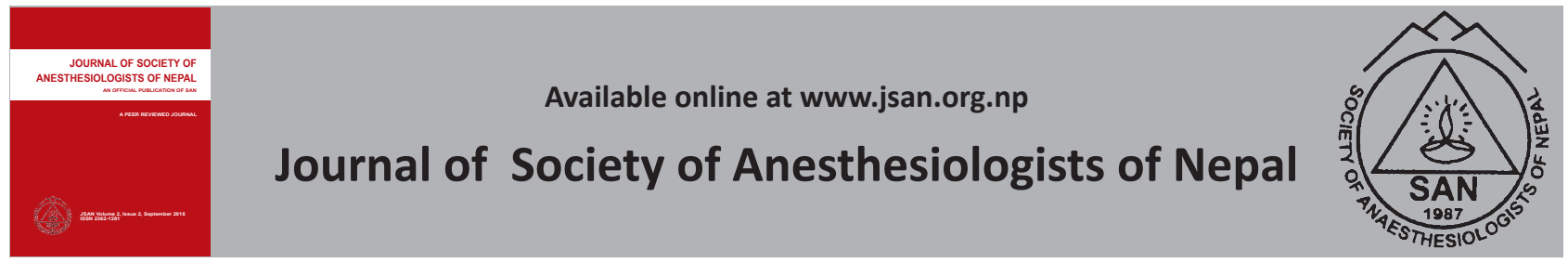

Case Report

\title{
Anaesthetic management of primary hyperparathyroidism with hypercalcaemia and multiple bone fractures for excision of parathyroid adenoma
}

\author{
Sabin Koirala ${ }^{\ddagger}$, Gentle Sunder Shrestha ${ }^{*}$

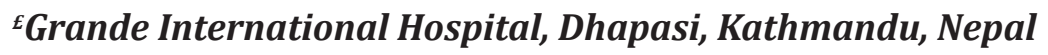 \\ ${ }^{\ddagger}$ Tribhuvan University Teaching Hospital, Institute of Medicine, Maharajgunj, Kathmandu, Nepal
}

\author{
ARTICLE INFO \\ Article History \\ Received 31.07.2015 \\ Accepted 23.08.2015 \\ Published 10.09. 2015 \\ (C) Authors retain copyright \\ and grant the journal right \\ of first publication with the \\ work simultaneously licensed \\ under a Creative Commons \\ Attribution License that allows \\ others to share the work with \\ an acknowledgement of the \\ work's authorship and initial \\ publication in this journal.
}

\begin{abstract}
Abstract: Calcium is important for maintaining numerous physiological processes many of which are important for anaesthesiologists. Primary hyperparathyroidism is an important cause of hypercalcaemia which can be associated with multitude of systemic problems. Our patient presented with complains of spontaneous fracture of bones, associated with pain in small joints since nine months. Later, she developed weakness all over the body and had obvious deformities in both extremities making her unable to perform her daily activities. Her total serum calcium, serum phosphorus and serum parathyroid hormone levels were elevated. She was diagnosed as severe hyperparathyroidism with hypercalcaemia, multiple bone fractures and deformities and was planned for excision of parathyroid adenoma. Management of hypercalcaemia was initiated preoperatively and was continued in intra-operative period. Meticulous attention was paid on patient positioning and prevention of further bone fractures. We used Dexmedetomidine to supplement anaesthetic agents, to facilitate smooth recovery and to prevent post-operative shivering. Close monitoring of electrocardiogram and neuromuscular function was done. Vigilant monitoring was done to detect possible postoperative complications, including hypocalcaemia. We present this case to discuss the complexity of problems that primary hyperparathyroidism can manifest and the related anaesthetic concerns.
\end{abstract}

Key-words: bone; fractures; hypercalcaemia; primary hyperparathyroidism

How to cite this article: Koirala S, Shrestha GS. Anaesthetic management of primary hyperparathyroidism with hypercalcaemia and multiple bone fractures for excision of parathyroid adenoma. JSAN 2015;2:87-90.

Corresponding author: Correspondences at:

Sabin Koirala, MD

Anaesthesiologist and Intensivist

Grande International Hospital, Dhapasi, Kathmandu, Nepal

Telephone: +9779851135841

Email: sabinkoirala@gmail.com 


\section{Introduction}

The elevation of serum calcium and inappropriate increase in the levels of parathyroid hormones constitute a constellation of symptoms of primaryhyperparathyroidism. ${ }^{1}$ The incidence of primary hyperparathyroidism is estimated to be 25 per 100000 general population. ${ }^{1}$ Most common cause is single parathyroid adenoma (80\%). ${ }^{1}$ The endocrine, electrolyte and metabolic disturbances resulting from such disorders can have a profound effect on the normal human physiological milieu. ${ }^{2}$ The most striking electrolyte disturbance during parathyroid surgery is the imbalance of calcium levels in the body and the main emphasis during the entire peri-operative period revolves around the maintenance of normal serum calcium levels. ${ }^{2}$ We here describe the successful anaesthetic management of a patient with primary hyperparathyroidism, who initially presented with hypercalcaemia and pathological fractures, to discuss the complexity of problems and their anaesthetic concerns.

\section{Case Report}

Thirty years old female presented with complains of spontaneous fracture of bones, associated with pain in small joints since nine months. Later, she developed weakness all over the body. She had obvious deformity in both extremities making her unable to perform daily activities. She had no other known diseases in the past which needed medical attention. There is no history of similar illness or other chronic or communicable diseases in her family members.

On examination, she was $132 \mathrm{~cm}$ in height and weighed 25 kilograms. She had obvious deformities in all four extremities. Range of motion in all the joints in all four extremities was limited. Both the lower limbs were bent above the ankle joint. There was a $2 \times 2 \mathrm{~cm}$ sized nodule on left side of neck, which was non-tender and moved with deglutition. Investigation reports revealed normal renal function. Her alkaline phosphatase was high (2991 IU/L). Her thyroid function test was normal. Her total serum calcium (3.2 mmol/L) and serum phosphorus (7.4 $\mathrm{mg} / \mathrm{dL}$ ) were elevated. Serum Albumin level was $3.4 \mathrm{~g} / \mathrm{dL}$. Twenty-four hour urine calcium was high $(12.3 \mathrm{mmol} / 24$ hour). Serum parathyroid hormone (PTH) level was high $(2525 \mathrm{pg} / \mathrm{ml})$ and serum vitamin $\mathrm{D}_{3}$ was low (14.9 ng/ $\mathrm{ml})$. Ultrasound of neck showed well defined lesion in left lower pole of thyroid $(2.6 \times 1.7 \times 1.8 \mathrm{~cm})$ suggestive of parathyroid adenoma. Parathyroid scintigraphy with $99 \mathrm{~m}$ Tc pertechnetate findings was consistent with parathyroid adenoma-left lower parathyroid gland. X-Rays of cervical and lumbo-sacral spine showed osteopenia. X-Ray of skull showed similar findings. X-Rays of both upper and lower limbs showed old unhealed fractures with cystic and lytic changes and with deformities (Figure 1 and 2). X-Ray Kidney, Ureter and Bladder (KUB) showed no renal calculi. ECG was normal.

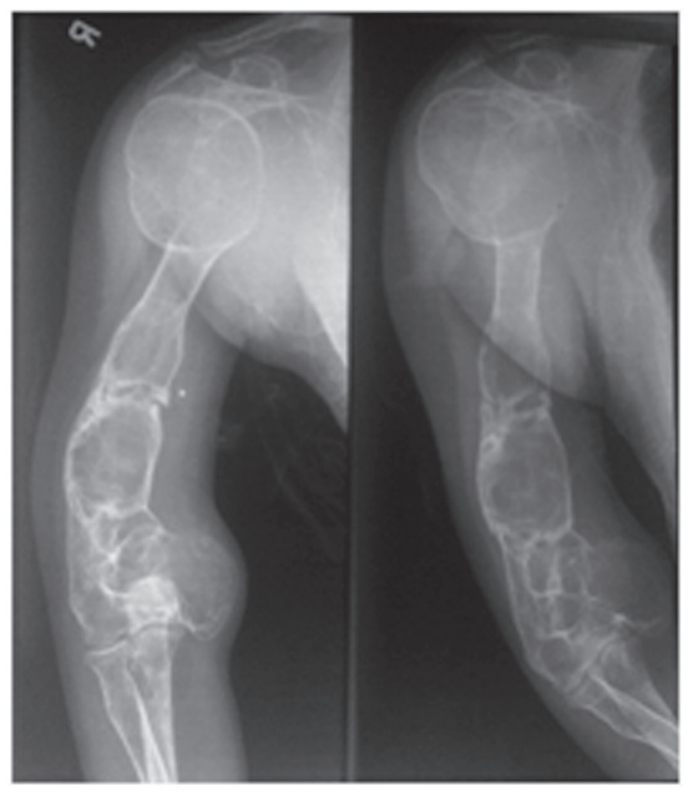

Figure 1: X-Ray of right humerus with right shoulder and elbow joint.

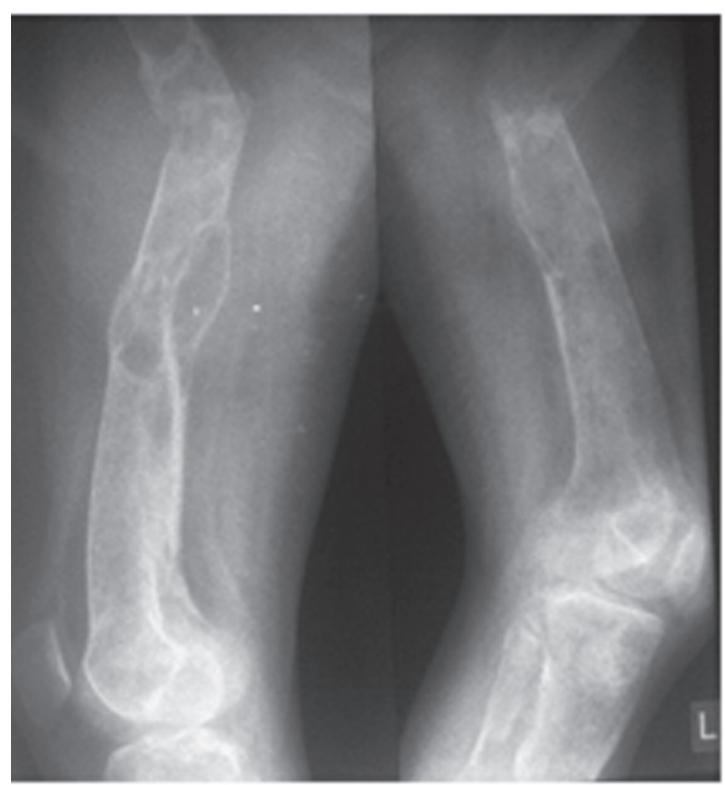

Figure 2: X-Rays of Femur bones (right and left) with knee joint

She was treated with adequate hydration, inj. Zoledronic acid $5 \mathrm{mg}$, followed by tab Alendronic acid $70 \mathrm{mg}$ once weekly. Skin traction was applied bilaterally for femur fracture. She was planned for excision of the parathyroid adenoma.

She was kept nil per oral for 8 hours. Normal Saline was continuously administered at $100 \mathrm{ml} / \mathrm{hr}$. Total Serum calcium was $2.9 \mathrm{mmol} / \mathrm{L}$ in the morning of surgery. In the operating room, monitoring with heart rate, ECG and 
pulse oximetry was started. Inj. dexmedetomidine was then started at a bolus dose of $1 \mathrm{mcg} / \mathrm{kg} / \mathrm{min}$ over 20 mins followed by infusion at $0.2 \mathrm{mcg} / \mathrm{kg} / \mathrm{hr}$. Left brachial artery was cannulated for invasive arterial blood pressure monitoring. Inj. Fentanyl $75 \mathrm{mcg}$ was given for analgesia. Induction of anaesthesia was done with Inj. propofol 50 $\mathrm{mg}$ and muscle relaxation for intubation was achieved with Inj Rocuronium 25 mg. Anaesthesia was maintained with oxygen, isoflurane at 0.6-0.9\% and dexmedetomidine at $0.4 \mathrm{mcg} / \mathrm{kg} / \mathrm{hr}$. Neuromuscular monitoring was done with Train of Four stimulation and maintenance of neuromuscular blockade was done with intermittent boluses of Rocuronium. Analgesia was supplemented with inj. paracetamol $1 \mathrm{gm} \mathrm{IV}$. After the excision of the mass, $20 \mathrm{ml}$ of $10 \%$ Calcium gluconate IV (in $100 \mathrm{ml} \mathrm{NS}$ ) was administered slowly over 15-20 minutes. Neuromuscular blockade was reversed and trachea was extubated. The position of the vocal cords was checked during extubation. Serum calcium level at PACU was $3.0 \mathrm{mmol} / \mathrm{l}$. Serial postoperative serum calcium level monitoring was done. It was $2.7 \mathrm{mmol} / \mathrm{l}$ at 6 hours, $2.8 \mathrm{mmol} / \mathrm{l}$ at 12 hours and $2.4 \mathrm{mmol} / \mathrm{l}$ at 24 hours. Serum PTH level 24 hours postoperatively and was normal $(9.5 \mathrm{pg} / \mathrm{ml})$. Oral calcium $1 \mathrm{gm} 6 \mathrm{hrly}$ was administered. It was followed by 12 hourly serum calcium monitoring for 3 days, which was between 2.1-2.4 $\mathrm{mmol} / \mathrm{l}$. Patient was discharged home on $5^{\text {th }}$ postoperative day with advice to continue oral Calcium Tablets and to follow-up in 1 week with serum calcium report.

\section{Discussion}

Calcium plays a central role in many physiological processes essential for life. Of these, processes that are more relevant to anaesthesiologists are the effects on myocardium, vascular smooth muscle, skeletal muscle and blood coagulation. It is the free extracellular calcium that mediates these effects, maintenance of which is affected by three main hormones: parathyroid hormone (PTH), Vitamin D, and Calcitonin. ${ }^{3}$ Action potentials in nerve and skeletal muscle can be explained almost entirely by sodium and potassium fluxes across the plasma membrane while calcium is required to mediate the excitation-contraction coupling in skeletal muscle. However myocardial contractility is proportional to the magnitude of an inward calcium current across the plasma membrane. This inward flux depends on the free extracellular calcium level. Increase in extracellular calcium levels can enhance myocardial contractility, hence the heart relaxes less during diastole and eventually stops in systole (calcium rigor) if the levels are excessively high.

Primary hyperparathyroidism is the most common cause of hypercalcaemia. If untreated, it can lead to many severe complications like osteitis fibrosa cystica and nephrocalcinosis. ${ }^{4}$ In symptomatic patients, the common findings include: renal calculi, bone pains, pathological fractures, muscle weakness or non-specific symptoms like depression. ${ }^{5}$ Cardiac manifestations can occur in the form of prolonged PR interval, short QT interval and systemic hypertension. ${ }^{3}$ Primary hyperparathyroidism is diagnosed when persistent hypercalcaemia is associated with increased parathyroid hormone level. ${ }^{1,6}$

Literatures are sparse about anaesthetic management of patients with primary hyperparathyroidism and hypercalcaemia. ${ }^{5} \quad$ Preoperative optimization of hypercalcaemia is important. This can be done with infusion of normal saline, phosphate or frusemide and use of bisphosphonates. ${ }^{2}$ Factors aggravating hypercalcaemia like dehydration and malnutrition should be avoided or treated. In this case we maintained adequate hydration and treated with bisphosphonates preoperatively. Continuous ECG monitoring is important as cardiac rhythm disturbances may occur. Neuromuscular monitoring is important as there can be unpredictable effect of neuromuscular blockers. ${ }^{5,7}$ In our case dosing of rocuronium was guided by train of four monitoring. Like in our patient, where fractures can occur even during measurement of blood pressure with non-invasive cuff, invasive arterial blood pressure monitoring may be beneficial. Methylene blue is sometimes used in cases when the localisation of the glands becomes difficult. However its use is losing popularity due to its adverse effects and its interference with the pulse oximeter reading. If used its dose should be limited to $5 \mathrm{mg} / \mathrm{kg}^{7}$ Meticulous attention for proper patient positioning is important to avoid harm. Special consideration should be made in positioning of neck and other body parts to prevent pressure sores and to minimize the risk of pathological fractures. ${ }^{7}$ Anaesthetic requirements may be reduced in symptomatic patients. Dexmedetomidine is commonly used in many setups which not only reduces the intraoperative requirements of anaesthetic agents but also facilitates smooth extubation and decreases the incidence of shivering. ${ }^{8,9}$ We used dexmedetomidine as an anaesthetic adjunct. Although Dexmedetomidine has shown to increase cytosolic calcium in astrocytes, this increase is independent of extracelluar calcium level. ${ }^{10}$ Bilateral recurrent laryngeal nerve injury induced by trauma or oedema can cause stridor and laryngeal obstruction due to unopposed adduction of vocal cords. It is good practice to check the position of the vocal cords during extubation to exclude recurrent laryngeal nerve damage. ${ }^{7}$ Vigilant monitoring for possible postoperative complications like bleeding, hypocalcemic tetany, recurrent laryngeal nerve injury, soft tissue injury and edema, respiratory obstruction, pain, and metabolic abnormalities is important. ${ }^{3}$ Severe Hypocalcemia may be clinically manifested by tetany, laryngeal spasms, bronchospasms, hypotension, cardiac arrhythmias, psychiatric manifestations or even seizures, and presence of Chvostek's and Trousseau's sign. Hypocalcemia can usually occur in the first 4 to 5 days postoperatively and then it normalizes. ${ }^{2,5}$ So serum calcium level should be checked regularly, at least at 6 hours and 24 hours postoperatively. ${ }^{7}$ Direct measurement of ionised calcium may be more useful but the measurement is limited by 
difficulties in accurate analysis, lack of standardization, and need for special handling, all resulting in increased cost. Patients can develop severe hypocalcemia and calcium therapy should be routinely initiated following parathyroidectomy. ${ }^{5}$ Oral supplementation $(<6 \mathrm{~g}$ of elemental calcium can be given daily) suffices for mild symptoms but severe hypocalcaemia requires intravenous calcium and magnesium. Severe postoperative hypocalcaemia may occur as a result of 'hungry bone syndrome' and hypomagnesaemia. In our case we monitored serum calcium for 3 days postoperatively and calcium therapy (intravenous) was initiated just after the removal of parathyroid adenoma and was converted to oral supplementation in the post-operative ward.

\section{Conclusion}

Primary hyperparathyroidism can have a multitude of associated problems. The endocrine, electrolyte and metabolic disturbances resulting from such disorder can have a profound effect on normal human physiology. The most striking electrolyte disturbance during parathyroid surgery is the imbalance of calcium levels and this is relevant to anaesthesiologists as calcium has effects on myocardium, vascular smooth muscle, skeletal muscle and blood coagulation. So, the main emphasis during the entire peri-operative period revolves around the maintenance of normal serum calcium levels, and also to prevent and manage complications caused by abnormal calcium levels.

\section{References}

1. Mithal R, Farndon JR. Parathyroid disease and calcium metabolism. Br J Anaesth 2000;85:29-43.

2. Bajwa S J S, Sehgal V. Anaesthetic management of primary hyperparathyroidism: A role rarely noticed and appreciated so far. Indian Journal of Endocrinology and Metabolism 2013;17:235-39.

3. Stoelting RK, Dierdorf SF. Endocrine diseases. In: Stoelting RK, Dierdorf SF (Eds). Anaesthesia and Co-existing disease, $4^{\text {th }}$ ed. Philadelphia: Churchill Livingstone;2002:421-5.

4. Aguilera M, Vaughan RS. Calcium and the anaesthetist. Anaesthesia 2000;55:779-90.

5. Chopra P, Mitra S. Patients with symptomatic primary hyperparathyroidism:An Anaesthetic Challenge. Indian J Anaesth 2009;53:492-95.

6. Kearns AE, Thompson GB. Medical and surgical management of hyperparathyroidism. Mayo Clinic Proc 2002;77:87-91.

7. Sasidharan P, Johnston I G. Parathyroid physiology and anaesthesia. Anaesthesia tutorial of the week 142. 2009.

8. Bajwa SJ, Gupta S, Kaur J, Singh A, Parmar SS. Reduction in the incidence of shivering with perioperative dexmedetomidine: A randomized prospective study. J Anaesthesiol Clin Pharmacol 2012;28:86-91.

9. Bajwa SS, Kaur J, Singh A, Parmar SS, Singh G, Kulshrestha A. et al. Attenuation of pressor response and dose sparing of opioids and anaesthetics with pre-operative dexmedetomidine. Indian J Anaesth 2012;56:123-8.

10. Chen Y, Zhao Z, Code WE, Hertz L. A correlation between dexmedetomidine-induced biphasic increases in free cytosolic calcium concentration and energy metabolism in astrocytes. Anesth Analg 2000;91:353-7. 